\title{
Blinded by the light: a nonhuman primate model of achromatopsia
}

\author{
Katherine E. Uyhazi and Jean Bennett \\ Center for Advanced Retinal and Ocular Therapeutics, F.M. Kirby Center for Molecular Ophthalmology, Scheie Eye Institute, University of Pennsylvania, Philadelphia, Pennsylvania, USA.
}

\begin{abstract}
Achromatopsia is an inherited retinal degeneration characterized by the loss of cone photoreceptor function. In this issue of the $J C I$, Moshiri et al. characterize a naturally occurring model of the disease in the rhesus macaque caused by homozygous mutations in the phototransduction enzyme PDE6C. Using retinal imaging, and electrophysiologic and biochemical methods, the authors report a clinical phenotype nearly identical to the human condition. These findings represent the first genetic nonhuman primate model of an inherited retinal disease, and provide an ideal testing ground for the development of novel gene replacement, gene editing, and cell replacement therapies for cone dystrophies.
\end{abstract}

\section{Color blindness}

On the remote South Pacific island of Pingelap, $10 \%$ of the population is completely color blind. Fishermen fish at night because they can see better in the moonlight. Affected families stay in cabins during the day and wear sunglasses and squint when they venture outside. Tropical sunlight is more of a curse than a paradise to these inhabitants, as bright light turns their black and white world into an overexposed blur that produces debilitating glare. Immortalized by Oliver Sacks in his 1997 book The Island of the Colorblind (1), the condition maskun - meaning "not see," now known as achromatopsia - is an inherited retinal disease resulting in the loss of function of cone photoreceptors. While achromatopsia affects approximately 1 in 50,000 people worldwide, its extremely high prevalence in Pingelap can be traced to a typhoon in 1775 that decimated the population and left only 20 sur- vivors, one of whom was a heterozygous carrier of the disease (2).

\section{Fifty shades of gray}

There are two main forms of achromatopsia in humans: complete achromatopsia (rod monochromacy or total color blindness), an autosomal recessive disease in which all three subtypes of cones are absent, and incomplete achromatopsia, a milder form in which one or more cone subtype has residual function. Unlike red-green color deficiency that affects up to eight percent of the male population, achromatopsia results in severely reduced visual acuity that often impairs the quality of life. Patients with complete achromatopsia typically present with decreased visual acuity ( 20/200), lack of color vision, abnormal repetitive eye movements, and severe photophobia (Figure 1). Because all three cone subtypes are dysfunctional, the retina relies on the exqui-

\section{Related Article: p. 863}

Conflict of interest: JB is a scientific (non-equity-holding) founder of Spark Therapeutics, and founder of CenSight Biologics, Limelight Bio, and Perch Therapeutics. She serves on scientific advisory boards for Acouos and Odylia Therapeutics and is the Principal Investigator of sponsored research agreements from Limelight Bio and Biogen. JB is a coinventor on a patent for a method to treat or slow the development of blindness, but waived any financial interest in this technology in 2002. JB is a coinventor on several other patents related to retinal gene therapy applications, as follows: 10,155,794; 9,567,376; 9,433,688; 9,249,425; 9,896,665; 8,147,823; 5,012,815; 20180369415; 20180369412; 20180153962; 20170319058; 201700143505; 20160263246. Reference information: J Clin Invest. 2019;129(2):513-515. https://doi.org/10.1172/JCI126205. sitely light-sensitive rod photoreceptors for both night- and daytime vision. However, rods are specialized to function well in dimly lit conditions, but are too sensitive to work efficiently in bright light, resulting in glare. Rods also have low spatial resolution, leading to decreased acuity.

There are currently six known causative genes of achromatopsia, almost all of which are components of the phototransduction cascade in cone photoreceptors (3). Approximately $75 \%$ of affected individuals have mutations in cyclic nucleotidegated channel beta 3 or alpha 3 (CNGB3 or CNGA3), while the remainder of cases are caused by mutations in the remaining four genes (GNAT2, PDE6C, PDE6H, or ATF6) $(3,4)$. There is currently no treatment for this condition. Patients are encouraged to wear visors and red-tinted glasses or contact lenses to minimize photophobia in bright environments. There are even wearable devices that allow patients to "hear colors" by converting color information into sound waves (5), but to date there are no approved therapies that improve visual acuity or color sensitivity.

\section{Monkey see, monkey do}

Much of our understanding of the pathophysiology of achromatopsia comes from small animal models of the disease, including Cnga3- and Cngb3-KO mice (6). One major limitation of these models, however, is that rodents have neither a specialized region of high-resolution vision (i.e., fovea) nor a cone photoreceptorenriched macula, and thus do not accurately represent the anatomy or function of the human central retina. The nonhuman primate eye closely resembles the human eye, but research efforts have been limited by the lack of available disease models and expense.

In this issue of the JCI, Moshiri and colleagues describe four related rhesus macaques at the California Primate Research Center with visual impairment and clinical findings consistent with 
A

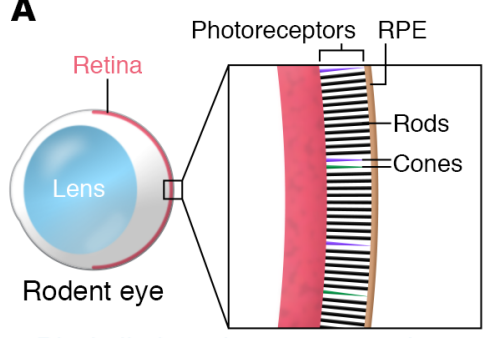

Dissimilarity to human eye makes biological research and interventional studies more difficult

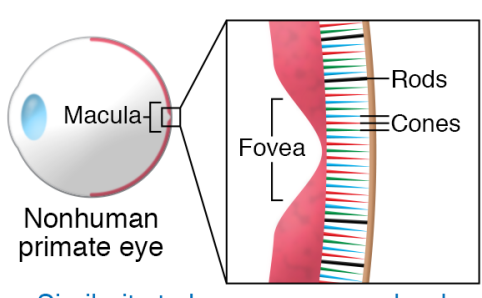

Similarity to human eye may lead to better biological research and interventional studies
B

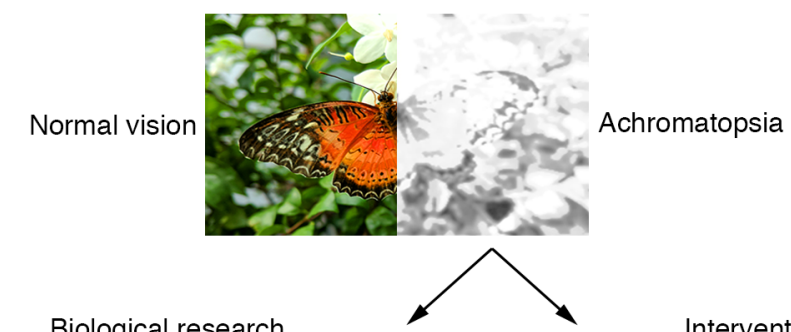

Biological research

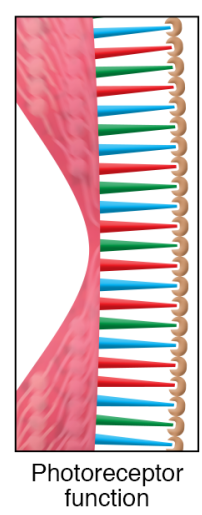

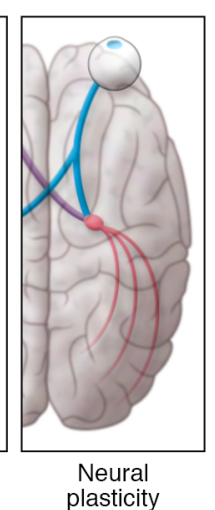

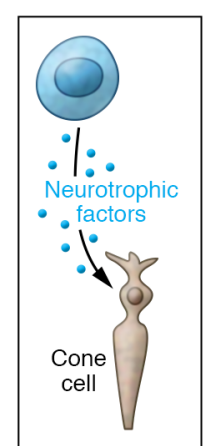

Neuroprotection
Interventional studies

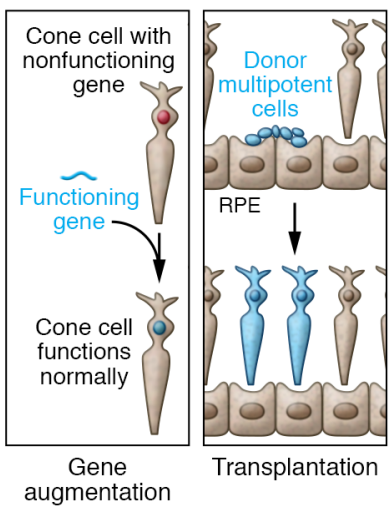

Figure 1. Complete achromatopsia typically results in poor visual acuity, lack of color vision, and severe photophobia due to inherited defects in cone photoreceptors. (A and B) Only primates have a macula, the region of the central retina enriched in cones and containing the cone-only fovea. Thus, availability of a nonhuman primate model will facilitate both basic research and interventional studies.

achromatopsia (7). The animals exhibited behavioral changes, including impaired navigation and tactile exploration of new environments that was worse in bright conditions such as outdoor daylight, reminiscent of the human disease. They had a pigmentary maculopathy, foveal thinning, and hyper- and hypoautofluorescent changes surrounding the fovea. Full-field scotopic and photopic ERG testing showed normal rod responses and absent cone responses. Whole-genome shotgun sequencing revealed homozygous missense mutations (R565Q) in the catalytic domain of the gene encoding cone phosphodiesterase 6C (PDE6C), a key component of the cone phototransduction cascade, which results in the expression of a catalytically inactive protein in vitro. Although mutations in PDE6C account for less than one percent of human achromatopsia (4), and this particular mutation has not been reported in humans, this model nonetheless represents a significant advance in the field as it shares a nearly identical phenotype with the human disease and provides a biologically relevant model of cone-mediated visual loss. Given the phenotypic similarities between the different molecular subtypes of achromatopsia in humans, this model may lend insight into the pathophys- iology of the more frequent CNGB3- and CNGA3-associated disease.

\section{An AAVenue towards a cure}

Achromatopsia represents an attractive target for gene therapy in humans for several reasons. First, the underlying genetics and causative mutations of the disease have been well characterized in multiple animal models of the disease. Second, cone cells remain physically present, albeit nonfunctional, in patients with achromatopsia and can be targeted with cone-specific promoters. Third, since cones are densely concentrated in the central macula, subretinal delivery of a viral vector would easily encompass the entire region necessary for functional improvement in central vision. Lastly, although gene therapy rescue in other animal models of achromatopsia showed better outcomes in younger animals compared with older animals $(8,9)$, the disease is relatively stationary in humans and shows no direct correlation with age or genotype (10), thus there may be a large window of opportunity for treatment.

Several groups have shown remarkable improvement in cone function using adeno-associated virus-mediated (AAVmediated) gene therapy in murine (11-13), canine (8), and sheep (14) models of the disease. Expression of a corrective copy of the gene improves retinal morphology, cone survival, inflammatory responses, electrophysiological testing, and cone-mediated visual behavior (15). Subsequently, several phase I/II human trials are in progress for CNGB3- and CNGA3-related disease (16). Interestingly, not all cone-specific promoters that rescue cone function in small animal models are effective in primates (17). Therefore, the model reported by Moshiri et al. represents an invaluable resource for vector optimization and preclinical trial testing. Since the macaque eye is relatively similar in size, structure, and immune environment to the human eye, this naturally occurring disease model provides a rare opportunity to test the toxicity and dosage titration of clinical vectors, as well as the structural and functional rescue of cone photoreceptor cells after gene therapy. It would be especially interesting to assess the immunogenicity of AAV treatments in these macaques, as the immunoprivileged status of the eye may be altered in inherited retinal conditions that involve retinal cell death, remodeling, and subsequent loss of the immune barriers normally found in healthy tissue.

This model also provides a platform to test neuroprotective agents and cell- 
based therapies designed to slow, prevent, or reverse photoreceptor cell death. Since these therapies are not gene specific, the broader utility of the model lies in testing strategies to treat photoreceptor loss in general. Even in rod-specific diseases, which account for the majority of retinal degenerative conditions, the ultimate loss of visual acuity in human blinding disorders results from the associated death of cone photoreceptors. Therefore, this model may be useful to understand rodcone interactions and to test cone protective strategies such as rod-derived cone viability factor (RdCVF) (18). If even a small percentage of cones could be preserved in humans, a significant improvement in vision is possible. However, not all treatments that improve cone function may translate into therapeutic targets. For example, ciliary neurotrophic factor (CNTF) can improve cone function in a canine model of CNGB3-associated achromatopsia, but results in a decline in rod function and no improvement in cone function when expressed intravitreally in human patients (19). Adaptive optics scanning light ophthalmoscopy, in which single photoreceptor cells can be imaged in vivo, may be useful to identify the earliest structural changes in the retina, explore the interactions between rods and cones, and measure a response to treatment.

Lastly, induced pluripotent stem cellderived (iPSC-derived) photoreceptor transplantation experiments and CRISPRbased gene editing are both promising avenues of retinal regeneration research that are well suited to test in such a naturally occurring genetic model of photoreceptor dysfunction. Much of the ocular regeneration field has moved toward using iPSCs as a source of retinal progenitor cells. It has been historically difficult to generate fully differentiated cells in vitro for use in transplantation experiments. However, it is now possible to generate iPSC-derived retinal organoids that contain light-sensitive photoreceptor precursors with early outer segments (20). Cell transplantation experiments in this model could address several outstanding questions in the field. What are the ideal cells to transplant? Can transplanted precursors differentiate in vivo into functional cones? Do transplanted photoreceptors form appropriate synaptic connections in degenerated retina, and how are these processes regulated? Is functional improvement due to cellular integration, fusion, or to soluble neurotrophic factors? Answers to these questions may further our understanding of achromatopsia and bring us one step closer to developing better treatments for retinal diseases.

\section{Acknowledgments}

This work is supported by the Center for Retinal Ocular Therapeutics, University of Pennsylvania Perelman School of Medicine, Research to Prevent Blindness, the Brenda and Matthew Shapiro Stewardship, the Robert and Susan Heidenberg Investigative Research Fund for Ocular Gene Therapy, the Paul and Evanina Bell Mackall Foundation Trust, The Pennsylvania Lions Sight Conservation, and the F.M. Kirby Foundation. We are grateful to Tomas S. Aleman for helpful comments and for reviewing this commentary.

Address correspondence to: Jean Bennett, Center for Advanced Retinal and Ocular Therapeutics, Department of Ophthalmology, Perelman School of Medicine, 310 Stellar-Chance Laboratories, University of Pennsylvania, 422 Curie Boulevard, Philadelphia, Pennsylvania 19104, USA. Phone: 215.898.0915; Email: jebennet@ pennmedicine.upenn.edu.

1. Sacks O. The Island of the Colorblind. New York, New York, USA: Vintage Books; 1996.

2. Winick JD, Blundell ML, Galke BL, Salam AA, Leal SM, Karayiorgou M. Homozygosity mapping of the achromatopsia locus in the Pingelapese. Am J Hum Genet. 1999;64(6):1679-1685.

3. Remmer MH, Rastogi N, Ranka MP, Ceisler EJ. Achromatopsia: a review. Curr Opin Ophthalmol. 2015;26(5):333-340.

4. Hirji N, Aboshiha J, Georgiou M, Bainbridge J, Michaelides M. Achromatopsia: clinical features, molecular genetics, animal models and therapeutic options. Ophthalmic Genet. 2018;39(2):149-157.
5. Alfaro A, Bernabeu Á, Agulló C, Parra J, Fernández E. Hearing colors: an example of brain plasticity. Front Syst Neurosci. 2015;9:56.

6. Roosing S, Thiadens AA, Hoyng CB, Klaver CC, den Hollander AI, Cremers FP. Causes and consequences of inherited cone disorders. Prog Retin Eye Res. 2014;42:1-26.

7. Moshiri A, et al. A nonhuman primate model of inherited retinal disease. J Clin Invest. 2019;129(2):863-874.

8. Komáromy AM, et al. Gene therapy rescues cone function in congenital achromatopsia. Hum Mol Genet. 2010;19(13):2581-2593.

9. Mühlfriedel R, et al. AAV-mediated gene supplementation therapy in achromatopsia type 2: preclinical data on therapeutic time window and long-term effects. Front Neurosci. 2017;11:292

10. Aboshiha J, et al. A prospective longitudinal study of retinal structure and function in achromatopsia. Invest Ophthalmol Vis Sci. 2014;55(9):5733-5743.

11. Alexander JJ, et al. Restoration of cone vision in a mouse model of achromatopsia. Nat Med. 2007;13(6):685-687.

12. Carvalho LS, et al. Long-term and agedependent restoration of visual function in a mouse model of CNGB3-associated achromatopsia following gene therapy. Hum Mol Genet 2011;20(16):3161-3175.

13. Pang JJ, et al. AAV-mediated cone rescue in a naturally occurring mouse model of CNGA3-achromatopsia. PLoS One. 2012;7(4):e35250

14. Banin E, et al. Gene augmentation therapy restores retinal function and visual behavior in a sheep model of CNGA3 achromatopsia. $M o l$ Ther. 2015;23(9):1423-1433.

15. Michalakis S, et al. Restoration of cone vision in the $\mathrm{CNGA}^{-/-}$mouse model of congenital complete lack of cone photoreceptor function. $\mathrm{Mol}$ Ther. 2010;18(12):2057-2063.

16. Hassall MM, Barnard AR, MacLaren RE. Gene therapy for color blindness. Yale J Biol Med. 2017;90(4):543-551.

17. Ye GJ, et al. Cone-specific promoters for gene therapy of achromatopsia and other retinal diseases. Hum Gene Ther. 2016;27(1):72-82.

18. Sahel JA, Léveillard T. Maintaining cone function in rod-cone dystrophies. In: Ash J, Anderson R, LaVail M, Bowes Rickman C, Hollyfield J, Grimm $\mathrm{C}$, eds. Retinal Degenerative Diseases. Advances in Experimental Medicine and Biology. Vol 1074. New York, New York, USA: Springer; 2018:499-509.

19. Zein WM, et al. CNGB3-achromatopsia clinical trial with CNTF: diminished rod pathway responses with no evidence of improvement in cone function. Invest Ophthalmol Vis Sci. 2014;55(10):6301-6308.

20. Zhong X, et al. Generation of three-dimensional retinal tissue with functional photoreceptors from human iPSCs. Nat Commun. 2014;5:4047. 